\title{
Scattering mechanisms, relaxation times, and shear viscosity in universal anomalous transport of unitary Fermi gases
}

\author{
Hang Zhou ${ }^{\circ}$, Hang Dong, and Yongli Ma ${ }^{*}$ \\ State Key Laboratory of Surface Physics and Department of Physics, Fudan University, Shanghai 200433, China
}

(Received 26 November 2019; accepted 27 April 2020; published 13 May 2020)

\begin{abstract}
In this paper we investigate the scattering mechanisms of viscous momentum transport in unitary Fermi gases below the pseudogap temperature $T^{*}$ by opening almost all the elastic and inelastic scattering channels. For a given system Hamiltonian, we adopt a microscopic $t$-matrix approximation to determine the thermodynamical quantities, and we approach an equivalent three-fluid dynamics to calculate the viscous relaxation time $\tau$ by two kinds of elementary excitations. The exotic scattering processes raised by uncondensed Fermi pairs greatly decay $\tau$ and lead to a universal behavior of $\tau$ with temperature, which is closely related to the anomalous transport in a wide range of strongly correlated systems. For linking our findings of $\tau$ to the shear viscosity $\eta$, we present a Kubo-based expression of $\eta$ by the stress-tensor correlation function with conserved vertex corrections, and we give an approximate relation for a possible experimental determination of $\tau$. Our results fit well with measurements and are comparable with other theories. Moreover, we verify the newly proposed universal upper bound of the ratio of $\eta$ and entropy density $s$ in unitary Fermi gases.
\end{abstract}

DOI: 10.1103/PhysRevResearch.2.023166

\section{INTRODUCTION}

Many strongly correlated systems display a remarkable degree of universal anomalous behaviors in their transport properties, such as the quantum-limited values of diffusivity for diverse fluids from quark-gluon plasma [1,2] to ultracold Fermi gases [3-5], and the linear-in-temperature $(T)$ resistivity in a wide range of materials [6]. Recent attention has linked these universalities to the relaxation time $\tau$, which typically carries fundamental coherence of corresponding transport and is highly associated with transport coefficients [7]. In particular, some studies have found that $\tau$ exhibits the same temperature dependence as the characteristic Planck time $\tau_{P}=\hbar /\left(k_{B} T\right)$ in the anomalous transport regions $[6,8,9]$ ( $\hbar=k_{B}=1$ hereafter), and some conjectures proposed that $\tau$ could lead to the universal behaviors of transport coefficients by controlling the universal lower and upper bounds [10,11].

For example, the well-known lower bound on the ratio of shear viscosity $\eta$ to entropy density $s$ conjectured by Kovtun et al. (KSS) [10], $\eta / s \geqslant 1 /(4 \pi)$, has motivated great interest in searching for a nearly perfect fluid, where the minimum of $\eta / s$ is obtained when $\tau$ is assumed to be equal to $\tau_{P}$. The shear viscosity is closely related to the transverse momentum diffusion coefficient $D_{\eta}=\eta /(s T)$ in a wide class of interacting systems. Lately, this bound has been generalized to any diffusion $D \gtrsim v^{2} / T$ with the typical velocity scale $v[8,9]$.

\footnotetext{
*ylma@fudan.edu.cn

Published by the American Physical Society under the terms of the Creative Commons Attribution 4.0 International license. Further distribution of this work must maintain attribution to the author(s) and the published article's title, journal citation, and DOI.
}

And from causality, an upper bound has also been proposed as $D \lesssim v^{2} \tau$ [11], which for the transverse momentum transport can be written as $\eta / s \lesssim v^{2} T \tau$, where $T \tau$ could be a constant for a $T$-linear scattering rate $\tau^{-1}=\alpha T$. Interestingly, besides the hydrodynamic fluids, analysis of many materials with $T$-linear resistivity revealed the phenomenological constant $0.9<\alpha<2.2$ in the anomalous $T$-linear region, despite the considerable differences in their microscopic natures [6].

It is tempting to establish a universal explanation for the robust transport behaviors. Before that, however, one should concretely investigate the basic scattering mechanisms in different strongly correlated systems. The low-frequency transport properties are controlled by the longest-lived excitations. In weakly coupled systems, the longest-lived excitations are the dominant quasiparticles. Equilibration in such systems is slow, and the relaxation time is much longer than the Planck time [11]. However, in strongly correlated systems, it is difficult to identify which excitations are dominant from the tight coupling of various energy states and environments, as their lifetimes may be affected by various exotic relaxation processes, and these additional degrees of freedom may invalidate some conventional transport laws.

The unique ability of highly tunable scattering length $a_{f}$ in ultracold Fermi gases makes the system an ideal platform to study the above properties systematically, which could undergo a smooth crossover from the BardeenCooper-Schrieffer (BCS) state of weakly correlated pairs of fermions to the Bose-Einstein condensation (BEC) of diatomic molecules $[12,13]$. In the vicinity of a Feshbach resonance, $a_{f} \rightarrow \infty$ is the so-called unitary Fermi gases (UFGs), which is a scale invariant, strongly interacting quantum manybody system. There are many measurements on the shear viscosity of UFGs $[3,14-16]$. At high temperatures, the kinetic temperature dependence $\eta \propto T^{3 / 2}$ [17] has been confirmed. 
Near the superfluid transition temperature $T_{c}$, the minimum value of $\eta / s$ turns out to be about several times the KSS bound. And in the superfluid phase $\eta$ behaves like superfluid ${ }^{3} \mathrm{He}$ without the upturn predicted by phonons $[18,19]$. The inhomogeneity caused by external traps in experiments can be circumvented by extracting the local data [16,20]. A quantum Monte Carlo (QMC) calculation of the frequencydependent shear viscosity from the imaginary time Kubo correlator meets an ill-posed problem and requires other theoretical constraints a priori [21-23]. Theoretical progress has proposed different constraints on viscosity spectral functions in the form of sum rules and high-frequency tails [24-28]. The static shear viscosity is the zero-frequency limit of the spectral functions. A calculation based on a self-consistent $t$-matrix approximation gives a careful result in the normal phase [25], and a calculation based on the pseudogap theory is consistent with the experimental results that $\eta$ decreases to zero in the superfluid phase [26]. As for the relaxation time $\tau$, an independent measurement of it is still lacking, which may be done in the same experiment for shear viscosity. Previous calculations of $\tau$ at high temperatures are accurate within the framework of kinetic theory [29], and the medium effects have been introduced into the scattering cross section at temperatures comparable to the Fermi temperature [30]. However, at the strong correlation and dissipation regimes near $T_{c}$, the fundamental properties of the system are only partially understood; thus the high- $T$ evaluation of $\tau$ is not applicable, and its intrinsic connection with $\eta$ is still ambiguous.

Viscous relaxation is a response to the transverse perturbation and is thus sensitive to the properties of excitations. It is an open question to open almost all the scattering channels among different excitations in the UFGs. In this paper, based on a pairing fluctuation theory which incorporates both fermionic and bosonic excitations, we present an equivalent three-fluid dynamics to investigate the scattering mechanisms in momentum transport. Due to the vanishing of bulk viscosity in the scale-invariant fluid [28,31,32], we show explicitly the dominant mechanisms of $\eta$ by various elastic and inelastic scatterings. The overall relaxation process exhibits a universal characteristic time behavior in the pseudogap regime above $T_{c}$, which results in an anomalously small $\eta$ [3] and contributes to broadened excitation spectra $[33,34]$. We then give an approximate relation connecting $\eta$ and $\tau$ with thermodynamic quantities, which is extended from an exact mean-field relation [35] based on the microscopic properties of pseudogap region. A generalized Kubo expression for $\eta$ is also carried out with conserved vertex corrections on stress-tensor correlation functions in the strong dissipation regimes. The results of $\eta$ yield fair agreement with measurements [16,20] and are comparable with other theories $[21,25,26]$. Finally, we verify the newly proposed universal upper bound of $\eta / s[11]$, which is related to $\tau$.

This paper is organized as follows. In Sec. II, we present a detailed description of the scattering processes. In Secs. II A and II B, we adopt the tractable microscopic theory called a "pseudogap" model to get the thermodynamical quantities and present an equivalent three-fluid dynamics to determine the effective scattering parameters. A main result of this work is the universal temperature dependence of relaxation time, which is shown in Sec. II C, as well as those detailed scattering mechanisms represented by the respective relaxation times. To verify our findings, we calculate the shear viscosity in Sec. III. The approximate relation of $\tau$ and $\eta$ are shown in Sec. III A, and the Kubo expressions for $\eta$ are in Sec. III B. They are compared with previous work in Sec. III C. Section IV concludes our work and gives a outlook.

\section{SCATTERING MECHANISMS}

We consider a 50-50 mixture of two Fermi species $\sigma=\uparrow, \downarrow$ in the BCS-BEC crossover scenario and start from the Hamiltonian with zero-range interactions [12]:

$$
\hat{H}=\sum_{\mathbf{p} \sigma} \xi_{\mathbf{p}} \hat{c}_{\mathbf{p} \sigma}^{\dagger} \hat{c}_{\mathbf{p} \sigma}+\frac{g_{f}}{2} \sum_{\mathbf{p} \mathbf{p}^{\prime} \mathbf{q} \sigma \sigma^{\prime}} \hat{c}_{\mathbf{p}+\mathbf{q} \sigma}^{\dagger} \hat{c}_{\mathbf{p}^{\prime}-\mathbf{q} \sigma^{\prime}}^{\dagger} \hat{c}_{\mathbf{p}^{\prime} \sigma^{\prime}} \hat{c}_{\mathbf{p} \sigma} .
$$

Here $\xi_{\mathbf{p}}=\mathbf{p}^{2} / 2 m_{f}-\mu$ is the dispersion measured from the chemical potential $\mu$, and $m_{f}$ is the mass of a Fermi atom. $\hat{c}_{\mathbf{p} \sigma}^{\dagger}\left(\hat{c}_{\mathbf{p} \sigma}\right)$ is the creation (annihilation) operator with the Fermi wave vector p. $g_{f}$ is the bare coupling strength characterized by $a_{f}$ at a given momentum cutoff $\Lambda, g_{f}=\frac{4 \pi a_{f}}{m_{f}} \frac{\pi}{\pi-2 \Lambda a_{f}}$. The density of Fermi atoms in volume $V$ is $n=\sum_{\mathbf{p} \sigma}\left\langle\hat{c}_{\mathbf{p} \sigma}^{\dagger} \hat{c}_{\mathbf{p} \sigma}\right\rangle / V$, where the average of operator $\hat{\mathcal{Q}}$ is performed by $\langle\hat{\mathcal{Q}}\rangle=$ $\operatorname{Tr}\left[e^{-\hat{H} / T} \hat{\mathcal{Q}}\right] / \operatorname{Tr}\left[e^{-\hat{H} / T}\right]$.

The stronger than BCS attractions arouse gap structures of the single-particle excitations in the normal state roughly at temperature $T^{*}$, known as the "pseudogap state," where additional incoherent bosonic excitations from the noncondensed fermion pairs $\left(b_{1}\right.$, density $\left.n_{b_{1}}\right)$ occur simultaneously with fermionic excitations $\left(f\right.$, density $\left.n_{f}\right)$. Below the superfluid phase transition temperature $T_{c}$, the condensation of pairs $\left(b_{0}\right.$, density $n_{b_{0}}$ ) occurs in the ordered phase, where the particlenumber conservation relation $n=n_{f}+2 n_{b}$ holds with $n_{b}=$ $n_{b_{0}}+n_{b_{1}}$. We may use "bosons" ( $b$, density $\left.n_{b}\right)$ to describe the fermion pairs hereafter.

In many cases, transport processes are governed by the intrinsic timescale $\tau$, which also corresponds to the lifetime of carriers. Its inverse, the relaxation rate $\tau^{-1}$, is often set as the characteristic energy scale to determine the boundary between the collisionless and hydrodynamic regimes. For UFGs, the system is well described by long-lived Fermi quasiparticles at temperatures well above the Fermi temperature $\varepsilon_{F}$. Kinetic theory gives the accurate result that $\tau \sim T^{1 / 2}$ [29], where the energy-dependent scattering cross section is independent of $a_{f}$. When the system enters the temperature regions comparable to or less than $\varepsilon_{F}$, medium effects have been introduced into the scattering cross section [30], which significantly decrease $\tau$. As $T<T^{*}$, the emergence of bosonic degrees of freedom changes the microscopic nature of UFGs; thus the modifications on the scattering cross section is not enough. According to the authors themselves, it is assumed that the strong interactions only modify the collision term while the streaming terms are unaffected [17].

In this section, focusing on temperature regimes $T \leqslant T^{*}$, we present an estimation of $\tau$ by the combination of microscopic theory with our equivalent three-fluid $\left(f, b_{0}, b_{1}\right)$ dynamics. By comprehensively considering various relaxation processes in different elementary components, we exhibit the 
universal behavior of $\tau$ that is responsible for many anomalous transports.

\section{A. Scattering processes}

For a particular elementary excitation $\varrho$ in a given fluid, its relaxation process can be generally regarded as the sum of all uncorrelated individual processes, denoted by scattering rate as $\frac{1}{\tau_{\varrho}}=\sum_{j l}\left(\frac{1}{\tau_{\varrho_{j l}}}+\frac{1}{\tau_{\varrho_{j l}}^{\prime}}\right)$. Here the subscript $j l$ denotes the incoming components in a reversible scattering process and the superscript / distinguishes the inelastic scattering. For the unitary Fermi gases, it microscopically consists of three elementary components $\left(f, b_{0}, b_{1}\right)$ in the superfluid phase [13], whose relaxation processes can be expressed in turn from Boltzmann or Gross-Pitaevskii equations, respectively $[30,36]$. The condensations $b_{0}$ have a considerably long dissipation time due to the signatures of phase coherence, but they do not participate in the momentum transport. The transport properties of the system are determined by the longer-lived excitation between $f$ and $b_{1}$, so here we consider $\varrho=(f, b)$ with the indices $j=\left(f, b_{1}\right), l=\left(f, b_{0}, b_{1}\right)$ and express the viscous relaxation processes by the Boltzmann equations as

$$
\begin{gathered}
\frac{\partial F}{\partial t}+\overrightarrow{\mathcal{F}}_{\text {ext }} \cdot \nabla_{\mathbf{p}} F+\frac{\mathbf{p}}{m_{f}} \cdot \nabla F=C[F], \\
\frac{\partial B}{\partial t}+\overrightarrow{\mathcal{F}}_{\text {ext }} \cdot \nabla_{\mathbf{q}} B+\frac{\mathbf{q}}{m_{b}} \cdot \nabla B=C[B] .
\end{gathered}
$$

$\overrightarrow{\mathcal{F}}_{\text {ext }}$ is the external force, and the mass of bosons $m_{b}$ is twice that of the fermions $m_{b}=2 m_{f}$. $\mathbf{q}$ is the Bose wave vector. $C[\mathcal{F}]=\sum_{j l}\left(C_{j l}[\mathcal{F}]+C_{j l}^{\prime}[\mathcal{F}]\right)$ is the total collision integral, where the inelastic scattering may be a composite boson split into two single fermions or its inverse process, and we have denoted $\mathcal{F}=F, B$ as the distribution functions for $n_{f}$ and $n_{b_{1}}$, which has the equilibrium correspondence $\mathcal{F}^{0}=B^{0}, F^{0}$ :

$$
F^{0}(\mathbf{p})=\frac{1}{e^{\left(\tilde{\varepsilon}_{\mathbf{p}}-\mu_{f}\right) / T}+1}, \quad B^{0}(\mathbf{q})=\frac{1}{e^{\left(\tilde{\varepsilon}_{\mathbf{q}}-\mu_{b}\right) / T}-1},
$$

and

$$
n_{f}=2 \sum_{\mathbf{p}} F^{0}(\mathbf{p}), \quad n_{b_{1}}=\sum_{\mathbf{q}} B^{0}(\mathbf{q}) .
$$

The chemical potentials of fermions $\mu_{f}$ and bosons $\mu_{b}$ satisfy the chemical equilibrium condition $\mu_{b}=2 \mu_{f}=2 \mu$, as the natural consequence of particle number conservation. The single-particle energy $\tilde{\varepsilon}_{\mathbf{p}(\mathbf{q})}=\varepsilon_{\mathbf{p}(\mathbf{q})}+u_{f(b)}$ is the sum of kinetic energy per particle $\varepsilon_{\mathbf{p}}=\mathbf{p}^{2} / 2 m_{f}\left(\varepsilon_{\mathbf{q}}=\mathbf{q}^{2} / 2 m_{b}\right)$ and the corresponding interaction energy $u_{f(b)}$ [see Eq. (10) below].

The Boltzmann equation can be variationally solved with errors less than $2 \%$ [30]. Under the relaxation time approximation, the elastic and inelastic scattering rates for a given process can be evaluated by

$$
\frac{1}{\tau_{\varrho_{j l}}}=\frac{-\left\langle\Psi^{*} C_{j l}[\mathcal{F}]\right\rangle_{0}}{\left\langle\Psi^{*}\left(\mathcal{F}-\mathcal{F}^{0}\right)\right\rangle_{0}}, \quad \frac{1}{\tau_{\varrho_{j l}}^{\prime}}=\frac{-\left\langle\Psi^{*} C_{j l}^{\prime}[\mathcal{F}]\right\rangle_{0}}{\left\langle\Psi^{*}\left(\mathcal{F}-\mathcal{F}^{0}\right)\right\rangle_{0}} .
$$

Here $\langle\cdots\rangle_{0}$ denotes the average under a trial function $\Psi$ with the linear change $\delta \mathcal{F}=\mathcal{F}-\mathcal{F}^{0}=\mathcal{F}^{0}\left(1 \pm \mathcal{F}^{0}\right) \Psi$ and $+(-)$ denotes bosons (fermions). The choice of trial function determines the specific type of relaxation, such as viscous, thermal, and conductive. We evaluate all elastic scattering and those inelastic scatterings whose contributions may not be ignored. They contribute to the relaxation rates of elementary excitation $\varrho=(f, b)$ as the following terms:

$$
\begin{aligned}
& \frac{1}{\tau_{\mathrm{f}}}=\frac{1}{\tau_{\mathrm{f}_{f f}}}+\frac{1}{\tau_{\mathrm{f}_{f b_{0}}}}+\frac{1}{\tau_{\mathrm{f}_{f b_{0}}}^{\prime}}+\frac{1}{\tau_{\mathrm{f}_{f b_{1}}}}+\frac{1}{\tau_{\mathrm{f}_{f b_{1}}}^{\prime}}, \\
& \frac{1}{\tau_{\mathrm{b}}}=\frac{1}{\tau_{\mathrm{b}_{f b_{0}}}}+\frac{1}{\tau_{\mathrm{b}_{f b_{1}}}}+\frac{1}{\tau_{\mathrm{b}_{f b_{1}}}^{\prime}}+\frac{1}{\tau_{\mathrm{b}_{b_{1} b_{0}}}}+\frac{1}{\tau_{\mathrm{b}_{b_{1} b_{1}}}} .
\end{aligned}
$$

The tedious expressions of the various collision integrals both for the elastic $C_{j l}[\mathcal{F}]$ and for the inelastic $C_{j l}^{\prime}[\mathcal{F}]$, which are used in Eq. (5) to calculate every term in Eq. (6), are performed in Appendix, where the additional $s$-wave scattering lengths of $a_{b}$ and $a_{f b}$ for boson-boson and fermion-boson scattering processes respectively will be determined in the next subsection.

Above is our first kind of elementary excitations for approaching the relaxation time of the system, by considering the respective relaxation times of fermions and composite bosons to find the longer one. We can also give the second kind of elementary excitation: since this is a single-component Fermi system, we can alternatively treat the elementary excitations of the system approximately as only fermions, while the composite bosons play a medium role in the three-fluid dynamics [36]. In this case, the overall relaxation rate is given by the following scattering channels:

$$
\begin{aligned}
\frac{1}{\tau} & =\frac{-\sum_{j l}\left\langle\Psi^{*}\left(C_{j l}[F]+C_{j l}^{\prime}[F]\right)\right\rangle_{0}}{\left\langle\Psi^{*}\left(F-F^{0}\right)\right\rangle_{0}} \\
& =\frac{1}{\tau_{f f}}+\frac{1}{\tau_{f b_{0}}}+\frac{1}{\tau_{f b_{0}}^{\prime}}+\frac{1}{\tau_{f b_{1}}}+\frac{1}{\tau_{f b_{1}}^{\prime}}+\frac{1}{\tau_{b_{1} b_{0}}}+\frac{1}{\tau_{b_{1} b_{1}}} .
\end{aligned}
$$

The expressions of the various collision integrals $C_{j l}[F]$ and $C_{j l}^{\prime}[F]$ are still performed in the Appendix for $\mathcal{F}=F$.

\section{B. Microscopic model and equivalent three-fluid dynamical model}

The thermodynamical quantities, such as $T_{c}, T^{*}, \mu$, energy gap $\Delta$, and single-particle energy $\varepsilon$, can be obtained by an arbitrary microscopic theory, and the most commonly used microscopic approaches incorporating pairing fluctuations in the normal state to treat the strong couplings are the manybody $t$-matrix theories $[12,13]$. We choose the one with an asymmetric $t$-matrix form sometimes called the $\left(G G_{0}\right) G_{0}$ scheme, which reports the second-order superfluid phase transition and consists with the BCS ground state in the BCS-BEC crossover. Note that $\hat{\mathcal{Q}}(\tau)=e^{\tau \hat{H}} \hat{\mathcal{Q}} e^{-\tau \hat{H}}$, with the imaginary time $\tau=\mathrm{i} t$. The generalized single-particle normal imaginary time Green's function is defined by $G(\mathbf{p}, \tau)=$ $-\left\langle T_{\tau} \hat{c}_{\mathbf{p} \sigma}(\tau) \hat{c}_{\mathbf{p} \sigma}^{\dagger}(0)\right\rangle$, and the paired anomalous imaginary time Green's function is $F(\mathbf{p}, \tau)=\left\langle T_{\tau} \hat{c}_{-\mathbf{p} \downarrow}(\tau) \hat{c}_{\mathbf{p} \uparrow}(0)\right\rangle$ with the time order operator $T_{\tau}$. We use the notation $P=\left(\mathrm{i} \omega_{n}, \mathbf{p}\right)$, $Q=\left(\mathrm{i} \Omega_{m}, \mathbf{q}\right)$ for fermionic and bosonic four momenta, respectively, and $\sum_{P(Q)}=T \sum_{\mathbf{p}(\mathbf{q})} \sum_{\mathrm{i} \omega_{n}\left(\Omega_{m}\right)}$. The bare Green's function is $G_{0}(P)=\left(\xi_{\mathbf{p}}-\mathrm{i} \omega_{n}\right)^{-1}$. The dressed Green's function $G(P)$ mediates the interactions between fermions in the $t$ matrix $t(Q)^{-1}=g_{f}^{-1}+\sum_{P} G(P) G_{0}(Q-P)$ with the 


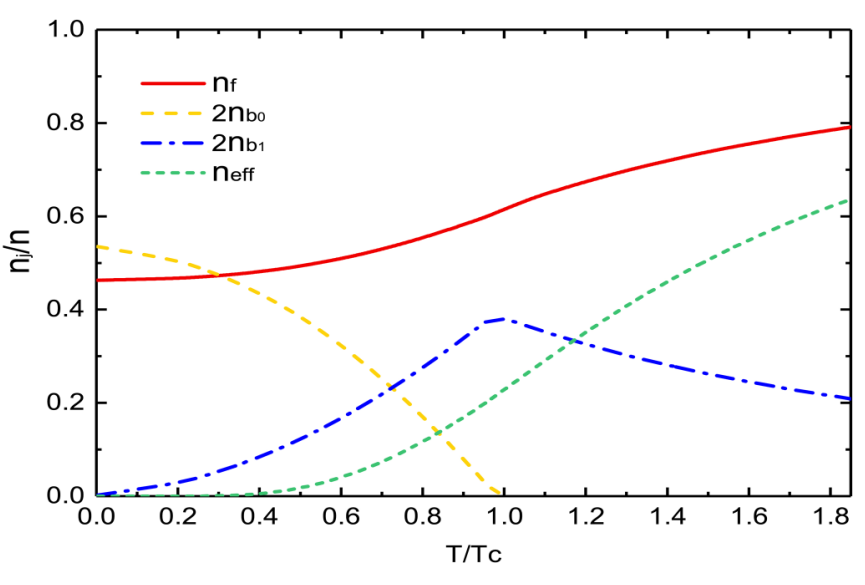

FIG. 1. Normalized particle densities: $n_{f}$ (red solid), $2 n_{b_{0}}$ (yellow dash), $2 n_{b_{1}}$ (blue dash dot), and $n_{\text {eff }}$ (green short dash) vs $T / T_{c}$.

regulation condition $\Lambda=\int d|\mathbf{p}|$. The self-energy is $\Sigma(P)=$ $\sum_{Q} t(Q) G_{0}(Q-P)$, which constructs $G(P)$ through the Dyson equation $G^{-1}(P)=G_{0}^{-1}(P)-\Sigma(P)$.

Based on the essential distinction between the total excitation-energy gap $\Delta$ and the superconducting order parameter $\Delta_{\text {sc }}$, one can adopt further simplifications to decompose $t(Q)$ into a standard BCS term $t_{\mathrm{sc}}(Q)=-\left(\Delta_{\mathrm{sc}}^{2} / T\right) \delta(Q)$ and the remaining nonzero momentum term $t_{\mathrm{pg}}(Q)=t(Q \neq$ 0 ), which leads to the pseudogap $\Delta_{\mathrm{pg}}$ approximately as $\Delta_{\mathrm{pg}}^{2} \equiv$ $-\sum_{Q \neq 0} t_{\mathrm{pg}}(Q)$. As a result, the total gap is also separated as $\Delta^{2}=\Delta_{\mathrm{sc}}^{2}+\Delta_{\mathrm{pg}}^{2}$.

In this pseudogap model, the densities of the elementary components (equivalent three-fluid effective dynamical quantities) are related to the gaps [13]

$$
\begin{aligned}
& n_{b_{0}}=Z \Delta_{\mathrm{sc}}^{2}, \\
& n_{b_{1}}=Z \Delta_{\mathrm{pg}}^{2}=\sum_{\mathbf{p}} \frac{1}{e^{\left(\mathbf{q}^{2} / 2 M^{*}-\mu_{\text {pair }}\right) / T}-1}, \\
& n_{f}=\sum_{\mathbf{p}} \frac{2}{e^{\left(\tilde{\varepsilon}_{\mathbf{p}}-\mu_{f}\right) / T}+1} .
\end{aligned}
$$

Here $Z$ is an expansion coefficient ensuring the particle number conservation $Z=\left(n-n_{f}\right) /\left(2 \Delta^{2}\right)$. This microscopic model gives almost the same form as Eq. (4), except that $u_{b}$ is absorbed into the effective pair mass $M^{*}$, resulting in an effective chemical potential $\mu_{\text {pair }}$ somewhat different from $\mu_{b}$. The three elementary components as functions of temperature are shown in Fig. 1.

At this point we can introduce an equivalent three-fluid dynamical model to determine the two unknown parameters $a_{b}$ and $a_{f b}$, which should be a function of the only independent interacting variable $a_{f}$ for the ultracold Fermi gases in the BCS-BEC crossover. The effective Hamiltonian density for given Eq. (1) is written as

$$
\begin{aligned}
\mathcal{H} & =\mathcal{H}_{0}+\mathcal{U}, \\
\mathcal{H}_{0} & =2 \sum_{\mathbf{p}} \varepsilon_{\mathbf{p}} F^{0}(\mathbf{p})+\sum_{\mathbf{q}} \varepsilon_{\mathbf{q}} B^{0}(\mathbf{q})+n_{b} \epsilon_{b}, \\
\mathcal{U} & =\frac{1}{4} g_{f} n_{f}^{2}+g_{b}\left(n_{b}^{2}-\frac{1}{2} n_{b_{0}}^{2}\right)+g_{f b} n_{f} n_{b} .
\end{aligned}
$$

$\epsilon_{b}$ is the dimer binding energy, and the coupling constants are $g_{b}=4 \pi a_{b} / m_{b}$ and $g_{f b}=4 \pi a_{f b} / m_{f b}$ with the reduced mass $m_{f b}^{-1}=m_{f}^{-1}+m_{b}^{-1}$. From $\mathcal{U}$, we can derive

$$
\begin{aligned}
& u_{b}=\frac{\partial \mathcal{U}}{\partial n_{b}}=2 g_{b} n_{b}+g_{f b} n_{f}, \\
& u_{f}=\frac{\partial \mathcal{U}}{\partial n_{f}}=\frac{1}{2} g_{f} n_{f}+g_{f b} n_{b} .
\end{aligned}
$$

Substituting Eq. (10) into Eq. (4), and equating $n_{f}, n_{b_{1}}$ in Eqs. (4) and (8), one obtains the undetermined coupling constants $g_{b}$ and $g_{f b}$. As a consequence, they are actually temperature dependent and can be viewed as two effective parameters. This is reasonable since the "bosons" at unitary are overlapping fermion pairs rather than tightly bound molecules, whose sizes and degrees of overlapping vary with temperatures. In addition, as we use the binary collisions to approach such a complex many-body problem, the encountered scattering cross sections must be regarded as effective ones. In other words, the many-body effects are considered in the effective scattering lengths. The precision of this effective approach is completely guaranteed by the microscopic theory, since the effective Hamiltonian density Eq. (9) is equivalent to the given system Hamiltonian Eq. (1).

\section{Results for the scattering mechanisms}

In Fig. 2, we exhibit the viscous relaxation times of elementary excitation $\varrho=(f, b)$, including the various individual processes expressed in Eq. (6). In Fig. 2(a), we find that $\tau_{\mathfrak{f}_{f f}}$ contributes the most to $\tau_{\mathrm{f}}$ at most temperature ranges, while $\tau_{\mathfrak{f}_{f b} b_{1}}$ acts as another dominant mechanism at the pseudogap regime. Near zero temperature, active fermions around the Fermi surface are condensed in pairs, and the remaining unpaired fermions hardly dissipate. An interesting comparison of $\tau_{\mathfrak{f}_{f b_{0}}}^{\prime}<\tau_{\mathfrak{f}_{f b_{0}}}$ shows the robustness of condensation: breaking the condensed pairs is even easier than exciting them. Circumstances in Fig. 2(b) are more complicated. $\tau_{\mathrm{b}}$ is strongly decayed by $n_{b_{0}}$ below $T_{c}$, corresponding to a continuous conversion from short-lived $n_{b_{1}}$ to long $n_{b_{0}}$. $\tau_{\mathrm{b}_{b_{1} b_{1}}}$ is not significant until near $T_{c}$, where the condensed pairs almost disappear. Above $T_{c}, \tau_{\mathrm{b}_{f b_{1}}}$ dominates as bosons become fermions.

The resulting $\tau_{\mathrm{f}}$ is always longer than $\tau_{\mathrm{b}}$, maintaining the basic Fermi nature of the UFGs. The overall relaxation time $\tau$ of the system should be close to $\tau_{\mathrm{f}}$. These results suggest the validity of our second kind of elementary excitation, the results of which are shown in Fig. 3(a). We can see that the scatterings between bosons give considerable impacts to $\tau$ near and below $T_{c}$ compared to $\tau_{\mathrm{f}}$, which is a reasonable consequence consistent with the physical nature. Our study is comprehensive and has included all the meaningful scattering channels: the elastic cases are complete since the scattering between condensed bosons $\tau_{b_{0} b_{0}}$ does not exist, and other inelastic cases can be ignored from the results that $\tau_{f b_{0}}^{\prime}$ and $\tau_{f b_{1}}^{\prime}$ take place on much longer timescales than their elastic counterparts outside the deep collisionless regime. This is sound, because pairing lowers the energy of fermions, which introduces certain constraints into incident particles to break bosons, thereby lowering the chance of inelastic collisions, 

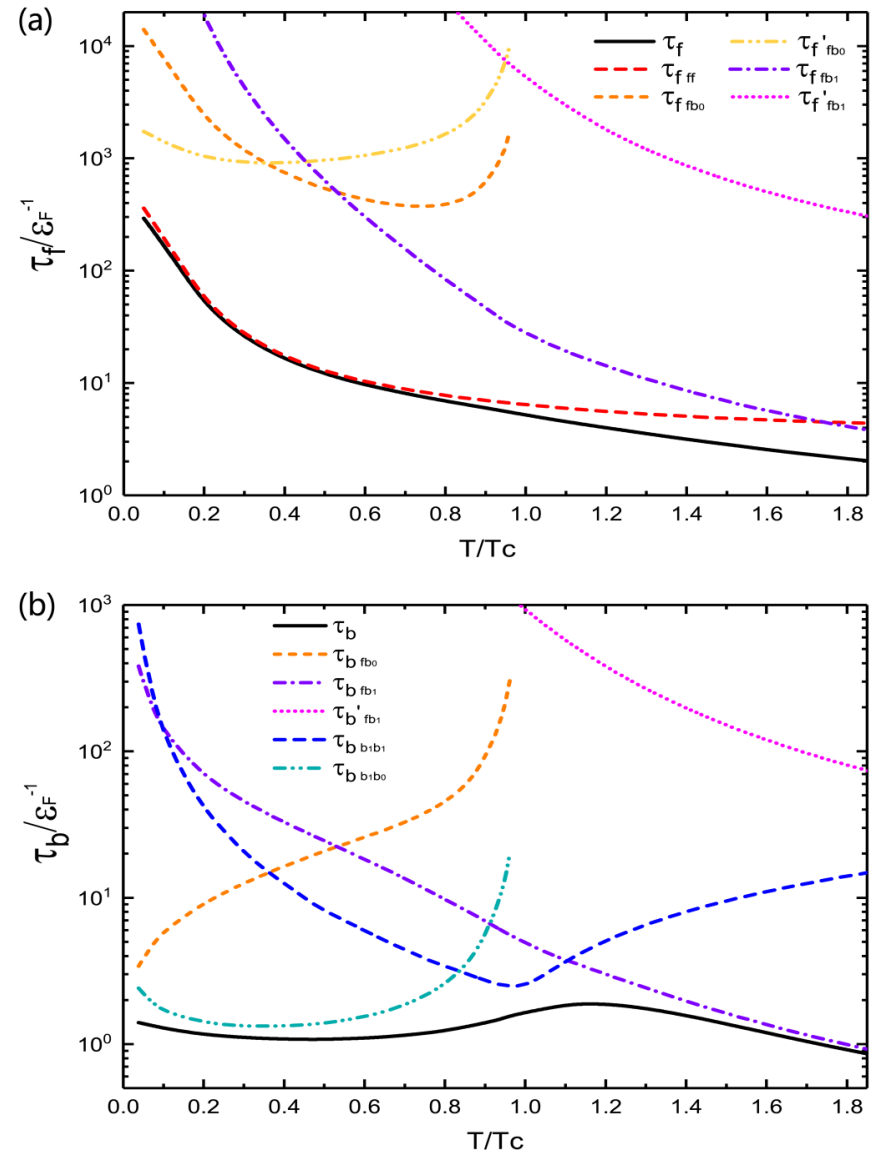

FIG. 2. Various viscous relaxation times normalized to the reciprocal of Fermi energy vs $T / T_{c}$ in the homogeneous UFGs. (a) Relaxation of unpaired fermions with the total lifetime $\tau_{f}$ (black solid), and components of $\tau_{\mathfrak{f}_{f f}}$ (red dash), $\tau_{\mathfrak{f}_{f b_{0}}}$ (orange short dash), $\tau_{\mathfrak{f}_{f b_{0}}}^{\prime}$ (yellow dash dot dot), $\tau_{\mathfrak{f}_{f b_{1}}}$ (purple dash dot), and $\tau_{\mathrm{f}_{f b_{1}}^{\prime}}^{\prime}$ (magenta dot). (b) Relaxation of uncondensed pairs with the total lifetime $\tau_{\mathrm{b}}$ (black solid), and components of $\tau_{\mathrm{b}_{b_{1} b_{1}}}$ (blue dash), $\tau_{\mathrm{b}_{f b_{0}}}$ (orange short dash), $\tau_{\mathrm{b}_{b_{1} b_{0}}}$ (cyan dash dot dot), $\tau_{\mathrm{b}_{f b_{1}}}$ (purple dash dot), and $\tau_{\mathrm{b}_{f b_{1}}}^{\prime}$ (magenta dot).

and the Pauli principle strongly inhibits the combinations of three fermions.

Above $T_{c}$, the fermion-boson scattering channel dominates in all three times, resulting in the departure from Fermi liquid theory and a similar temperature dependence with the Planck time $\tau_{P}$. We fit $\tau$ by $\tau=1 /(\alpha T)$ in Fig. 3(b) as a gray dashed line, with $\alpha \simeq 0.97$. Across a wide range of strongly correlated systems, the phenomenological dimensionless constant is $0.9<\alpha<2.2$ in the anomalous transport regimes [6], which shows the dramatic universality of the UFGs due to the pseudogap effects. The approximate lifetime of uncondensed pairs $\tau_{\mathrm{b}}$ is much shorter than the thermalization timescale $\tau$, which may explain why these pairs play no role in the equilibrium thermodynamics [37] of the system.

Since the relaxation rate $\tau^{-1}$ is often set as the characteristic energy scale of many collective oscillation modes, a continuous crossover from hydrodynamic $\left(\tau^{-1} \lesssim \varepsilon_{F}\right)$ to collisionless $\left(\tau^{-1} \ll \varepsilon_{F}\right)$ regimes arises smoothly as the temperature approaches zero, which has been observed recently [4]. The rapid rise of $\tau$ reveals a wide collisionless dynamic
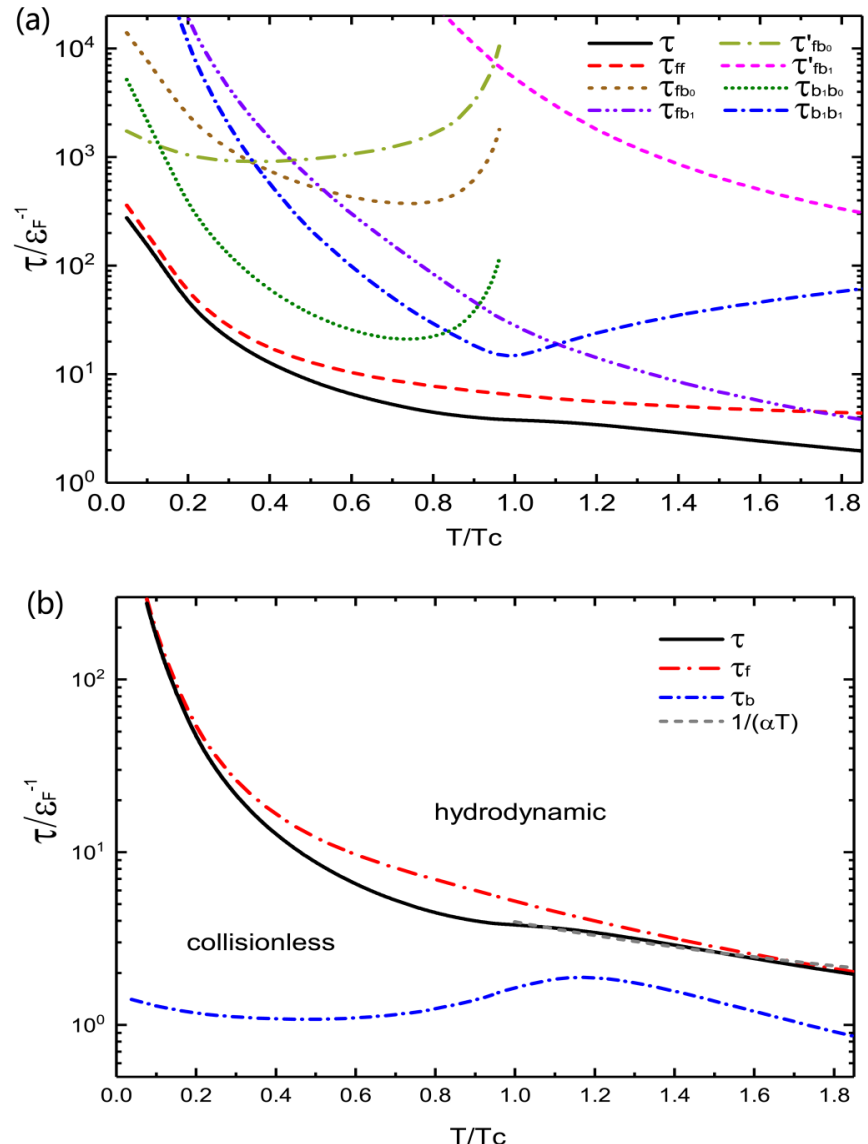

FIG. 3. Various viscous relaxation times vs $T / T_{c}$. (a) The total relaxation time $\tau$ (black solid), and the various relaxation channels $\tau_{f f}$ (red dash), $\tau_{b_{1} b_{1}}$ (blue short dash dot), $\tau_{b_{1} b_{0}}$ (olive short dot), $\tau_{f b_{0}}$ (brown dot), $\tau_{f b_{0}}^{\prime}$ (kelly dash dot), $\tau_{f b_{1}}$ (purple dash dot dot), and $\tau_{f b_{1}}^{\prime}$ (magenta short dash). (b) The total relaxation time of the system $\tau$ (black solid), fermions lifetime $\tau_{f}$ (red dash dot), and uncondensed bosons lifetime $\tau_{\mathrm{b}}$ (blue short dash dot). The gray dashed line is the fit of $\tau$ with $1 /(\alpha T)$.

regime where the second sound in two-fluid hydrodynamic descriptions rapidly damps into a diffusive mode, which is also consistent with the vanishing of the second sound peaks in observations $[4,38]$ and predictions based on phonon excitations $[39,40]$.

Finally, the temperature dependence of $\tau_{b}$ shows significant concavo-convex variations, which is caused by the alternations of dominant scattering mechanisms in our study. This trend provides an opportunity to examine our detailed calculations experimentally. Recent realizations of trapping UFGs at uniform density $[4,41]$ ensures the trend will not be hidden in the cloud averages. When this nonmonotonic behavior is observed, it will be a strong evidence of our results. Since measuring the two relaxation timescales $\tau_{f}$ and $\tau_{b}$ is by no means a trivial task, one can alternatively measure the decay times by exponential fitting of the displacements of $n_{b_{1}}$ and $n_{f}$ after a long evolution time, which also characterizes the momentum transfer to equilibrium. And in the spin transport measurements of UFGs, this timescale leads to the subsequent transport coefficients [5]. The remaining difficulty is to separate the three elementary components, where $n_{b_{0}}$ has 
been filtered out [4,42], and recent achievements such as the pair-breaking excitations [43] and quantum depletion in BoseEinstein condensates [44] can give prospects of distinguishing different excitations in momentum space.

\section{SHEAR VISCOSITY}

The experimental extraction of the intrinsic relaxation time $\tau$ is a challenging task which may be implemented modeldependently from, for example, the radio frequency excitation spectra as the lifetime of quasiparticles $[26,45]$. The situation will be difficult near $T_{c}$ because the spectral shape is not Lorentzian. A more direct means is to use a simple relation connecting $\tau$ with experimental observables. UFG is a scaleinvariant fluid due to the divergent $s$-wave scattering lengths; therefore the kinetic relation between the shear viscosity $\eta$ and the pressure $\mathcal{P}$ that $\eta=\mathcal{P} \tau$ is shown to apply in the normal phase [1]. Below $T_{c}$, an altered relation has been derived from the mean-field theory [35], with $\eta=\left(\mathcal{P}-\frac{2}{5} \mu n_{s}\right) \tau$, where $n_{s}$ is superfluid density, and the Cooper-pair contributions have been included on both sides. The kinetic relation has recently been implemented in experiments to test the reliability of using a hydrodynamic linear response [41]. From a theoretical perspective, pressure can be obtained by the minus density of the grand potential, and for UFGs it relates to the energy $\varepsilon$ with the relation $\mathcal{P}=2 \varepsilon n / 3$. At the moderate temperatures of interest in this paper, pairing fluctuations are significant and $\eta$ can be derived by the Kubo formula as the zero-frequency limit of the linear response function, which is highly related with $\tau$. Therefore, we can check the universal temperature dependence of $\tau$ alternatively by calculating $\eta$ and comparing it with measurements.

In the remainder of this section, first we present an approximate relation connecting $\eta$ and $\tau$ with thermodynamics, which is specialized for the pseudogap and superfluid regimes and provides an opportunity for experiments to estimate $\tau$. It automatically returns to the kinetic relation above $T^{*}$. Then we give a generalized Kubo-based expression of $\eta$ where $\tau$ lies implicitly in the self-energy and results in the broadened spectral functions. Both of the two new relations are smoothly applicable from zero- to high-temperature limits.

\section{A. Relation with thermodynamical quantities}

The kinetic relation $\eta=\mathcal{P} \tau$ is fulfilled only in the hightemperature limit where quasiparticles are well defined in the weak dissipation regime. A hypothetical form of viscosity spectral function exhibits that $\mathcal{P}$ is the Drude weight at zero frequency [25] and thus extrapolates the high- $T$ relation to smaller temperatures near $T_{c}$. A recent extraction of $\tau$ based on this relation did not capture the universal behaviors [40]. This may be the consequence of not sufficiently incorporating the pseudogap effects, which affect both the collision and streaming terms [17], and results in a considerably different microscopic nature.

We analyze the viscosity relation in an alternative way from the Einstein relation $\eta=\rho_{n} D_{\eta}$, with the normal fluid density $\rho_{n}=m_{f} n_{n}$ and the diffusivity $D_{\eta} \sim v^{2} \tau$. The high- $T$ relation $\eta=\mathcal{P} \tau$ comes from the estimate that $n_{n}=n$ and $m_{f} v^{2} n \sim \mathcal{P}$. In the pseudogap state, however, the additional bosonic degrees of freedom reduce the many-body effective carrier density $n_{\text {eff }}$ [26], which plays the role of normal density $n_{n}$ and is temperature dependent. Therefore, there should be some deviation of the viscosity-pressure relation between the pseudogap regime and the high- $T$ limit. Inspired by the low$T$ relation derived from mean-field theory [35], we give an approximate relation specialized at $T \leqslant T^{*}$ :

$$
\eta=\left[\frac{2}{3} \varepsilon n-\frac{2}{5} \mu\left(n-n_{\mathrm{eff}}\right)\right] \tau,
$$

where

$$
n_{\mathrm{eff}}=n-\frac{2}{3} \sum_{\mathbf{p}} \frac{\Delta^{2}}{E_{\mathbf{p}}^{2}} \frac{\mathbf{p}^{2}}{m_{f}}\left[\frac{1-2 F^{0}\left(E_{\mathbf{p}}\right)}{2 E_{\mathbf{p}}}+\frac{\partial F^{0}\left(E_{\mathbf{p}}\right)}{\partial E_{\mathbf{p}}}\right],
$$

with $E_{\mathbf{p}}=\sqrt{\xi_{\mathbf{p}}^{2}+\Delta^{2}}$.

The effective normal density $n_{\text {eff }}$ has been shown in Fig. 1 as short dashed green line, which has a different temperature dependence from $n_{f}$, like the situation between the superfluid density $n_{s}$ and $n_{b_{0}}$ in strongly correlated quantum fluids. At $T=0, n_{\mathrm{eff}}=0$ and the remaining part in the square brackets in Eq. (11) is consistent with the zero-temperature thermodynamic relation $\varepsilon=\frac{3}{5} \mu$, which ensures the disappearance of $\eta$ at low $T$ as observed experimentally [3,16]. This also explains why there is no contribution of superfluid phonons in $\eta$ [26]. Although the calculations of $\tau$ based on phonons also predict the collisionless regime near zero temperature [40], the upturn predictions of $\eta[18,39]$ cannot be observed. Our relation introduces the pseudogap effects by the replacement $n_{s} \rightarrow n-n_{\text {eff }}$ to go beyond the superfluid regime, and it will automatically return to the kinetic relation above $T^{*}$ when $n_{\text {eff }}=n$, whereas the beyond mean-field version given in the original literature $\eta=\left(\mathcal{P}-\frac{2}{5} \mu n\right) \tau$ [35] seems unable to do so.

It is worth noting that the relation of Eq. (11) is general and independent of the specific expression of $n_{\mathrm{eff}}$. Equation (12) is just a kind of estimate we choose to make the calculation of $\eta$ feasible under the unified framework of the pseudogap model. A more interesting extension of this relation may be an unknown two-fluid hydrodynamics in the anomalous regimes of a normal phase, with the new low-energy effective two-fluid components $n_{\text {eff }}$ and $n-n_{\text {eff }}$, which for the moment we may call "pseudo-two-fluid" dynamics. The existence of different effective carriers is a pervasive feature in the nonspontaneous symmetry-breaking state of many materials [6]. Moreover, the measurements of $n_{\text {eff }}$ may be accessible based on new dynamics, like the measurements of $n_{s}$ by Landau hydrodynamics [38], and which also enable the qualitative estimation of $\tau$ by Eq. (11).

\section{B. Kubo expression from stress-tensor correlator}

Strictly speaking, the viscosity-thermodynamics relation in the former section is an extrapolation of both low- and hightemperature limits, which implies use of the constraint $\tau^{-1} \ll$ $\varepsilon_{F}$, which is only valid in the weak dissipation regimes. To verify its validity at moderate temperatures of the strong dissipation regimes, we present a generalized Kubo expression with conserved vertex corrections from the stress-stress correlator within the pseudogap model. 
Formally, $\eta$ is defined as the static limit of the corresponding viscosity spectral function in terms of the imaginary part of the stress-tensor correlation function

$$
\eta=-\lim _{\Omega \rightarrow 0}\left\{\frac{\operatorname{Im} \Xi_{x y, x y}^{\mathrm{ret}}(\mathbf{q}=\mathbf{0}, \Omega)}{\Omega}\right\} .
$$

The retarded correlation function $\Xi_{x y, \bar{x} \bar{y}}^{\mathrm{ret}}(\mathbf{q}, \Omega)$ is Fourier transformed from the real-time response

$$
\Xi_{x y, \bar{x} \bar{y}}^{\mathrm{ret}}(\mathbf{q}, \Omega)=-\mathrm{i} \int d t e^{\mathrm{i} \Omega t} \Theta(t)\left\langle\left[\hat{\Pi}_{x y}(\mathbf{q}, t), \hat{\Pi}_{\bar{x} \bar{y}}(-\mathbf{q}, 0)\right]\right\rangle,
$$

where the stress tensor $\hat{\Pi}_{x y}$ contributing to $\eta$ involves only the off-diagonal bilinear kinetic term [25,28]

$$
\hat{\Pi}_{x y}(\mathbf{q}, t)=\sum_{\mathbf{p} \sigma} \Gamma_{x y}^{0}(\mathbf{p}, \mathbf{p}+\mathbf{q}) \hat{c}_{\mathbf{p} \sigma}^{\dagger}(t) \hat{c}_{\mathbf{p}+\mathbf{q} \sigma}(t),
$$

with $\Gamma_{x y}^{0}(\mathbf{p}, \mathbf{p}+\mathbf{q})=(2 \mathbf{p}+\mathbf{q})_{x}(2 \mathbf{p}+\mathbf{q})_{y} /\left(4 m_{f}\right)$ being the bare vertex factor.

The Matsubara correlation function $\Xi_{x y, x y}(Q)$ can be represented as a bubble diagram in terms of the product of two Green's functions in the form

$$
\begin{aligned}
\Xi_{x y, x y}(Q)= & \sum_{P} \Gamma_{x y}^{0}(\mathbf{p}, \mathbf{p}+\mathbf{q}) G(P) G(P+Q) \\
& \times \Gamma_{x y}(P+Q, P),
\end{aligned}
$$

where the dressed vertex function $\Gamma_{x y}$ can be given by an integral equation on the $t$-matrix level,

$$
\begin{aligned}
\Gamma_{x y}(P+Q, P)= & \Gamma_{x y}^{0}(\mathbf{p}+\mathbf{q}, \mathbf{p})+\sum_{Q^{\prime}} t\left(Q^{\prime}\right) G\left(Q^{\prime}-P\right) \\
& \times G\left(Q^{\prime}-P-Q\right) \Gamma_{x y}\left(Q^{\prime}-P-Q, Q^{\prime}-P\right) .
\end{aligned}
$$

For $\Gamma_{x y}$, the Ward identities can be maintained by three types of ladder diagrams in the $\left(G G_{0}\right) G_{0}$ scheme: a direct contribution called the Maki-Thompson (MT) diagram, and two exchange contributions called Aslamazov-Larkin (AL) diagrams [46]. In the same spirit of pseudogap approximations, by decomposing $t\left(Q^{\prime}\right)$ into two parts, $\Gamma_{x y}$ can be decomposed into the superconducting $\Gamma_{\mathrm{sc}}$ and pseudogap $\Gamma_{\mathrm{pg}}$ contributions (omitting the subscript $x y$ ), where the former contains only the MT diagram, as $\Gamma=\Gamma^{0}+\Gamma_{\mathrm{sc}}^{\mathrm{MT}}+\Gamma_{\mathrm{pg}}^{\mathrm{MT}}+\Gamma_{\mathrm{pg}}^{\mathrm{AL} 1}+\Gamma_{\mathrm{pg}}^{\mathrm{AL} 2}$. Moreover, they satisfy a further simplified relation $\Gamma_{\mathrm{pg}}^{\mathrm{AL} 1}+$ $\Gamma_{\mathrm{pg}}^{\mathrm{AL} 2}=-2 \Gamma_{\mathrm{pg}}^{\mathrm{MT}}[46]$.

One then inserts Eq. (17) with these diagrammatic contributions into Eq. (16), and it turns to a compact form,

$$
\begin{aligned}
\Xi_{x y, x y}(Q)= & \sum_{P} \Gamma_{x y}^{0}(\mathbf{p}, \mathbf{p}+\mathbf{q}) \Gamma_{x y}^{0}(\mathbf{p}, \mathbf{p}-\mathbf{q}) \\
& \times\left[G(P) G(P+Q)-F_{\mathrm{sc}}(P) F_{\mathrm{sc}}(P+Q)\right. \\
& \left.+F_{\mathrm{pg}}(P) F_{\mathrm{pg}}(P+Q)\right] .
\end{aligned}
$$

After doing the frequency summation, the analytical continuation $\mathrm{i} \Omega_{m}=\Omega+\mathrm{i}^{+}$, and taking the limit $\Omega \rightarrow 0$ in Eq. (13), we obtain the compact final form:

$$
\begin{aligned}
\eta= & -\frac{1}{15 m_{f}^{2}} \int \frac{d^{3} \mathbf{p}}{(2 \pi)^{3}} \mathbf{p}^{4} \int \frac{d \epsilon}{2 \pi} \frac{\partial F^{0}(\epsilon)}{\partial \epsilon} \\
& \times\left[A^{2}(\mathbf{p}, \epsilon)-B_{\mathrm{sc}}^{2}(\mathbf{p}, \epsilon)+B_{\mathrm{pg}}^{2}(\mathbf{p}, \epsilon)\right] .
\end{aligned}
$$

Here the spectral functions of the generalized normal and anomalous Green's functions $A(\mathbf{p}, \epsilon)=-2 \operatorname{Im} G(\mathbf{p}, \epsilon)$ and $B_{\mathrm{sc}(\mathrm{pg})}(\mathbf{p}, \epsilon)=-2 \operatorname{Im} F_{\mathrm{sc}(\mathrm{pg})}(\mathbf{p}, \epsilon)$ are the same as those of the pseudogap model and some high- $T_{c}$ literature $[27,45]$ :

$$
\begin{aligned}
G(\mathbf{p}, \omega) & =\left(\omega-\xi_{\mathbf{p}}+\mathrm{i} \gamma-\frac{\Delta_{\mathrm{pg}}^{2}}{\omega+\xi_{\mathbf{p}}+\mathrm{i} \gamma}-\frac{\Delta_{\mathrm{sc}}^{2}}{\omega+\xi_{\mathbf{p}}}\right)^{-1}, \\
F_{\mathrm{sc}}(\mathbf{p}, \omega) & =\frac{-\Delta_{\mathrm{sc}}}{\omega+\xi_{\mathbf{p}}+\mathrm{i} 0^{+}} \frac{1}{\omega-\xi_{\mathbf{p}}-\frac{\Delta^{2}}{\omega+\xi_{\mathbf{p}}}+\mathrm{i} 0^{+}} \\
F_{\mathrm{pg}}(\mathbf{p}, \omega) & =\frac{-\Delta_{\mathrm{pg}}}{\omega+\xi_{\mathbf{p}}+\mathrm{i} \gamma} G(\mathbf{p}, \omega) .
\end{aligned}
$$

The additional term $\gamma=(2 \tau)^{-1}$ is inversely proportional to $\tau$, which acts as a non-negligible damping term in the imaginary part of retarded self-energy, leading to the broadening of the spectral functions. Such broadening is a universal feature that also shows incoherent metals with $T$-linear resistivity $[8,11]$. Note that [28] did a great job in computing the nonperturbative results for the frequency-dependent shear viscosity spectral function by including the self-energy, MT, and AL diagrams in the stress-tensor correlation functions and applying them to high-temperature regimes. Our calculations mainly deal with the fundamental natures at $0 \leqslant T \lesssim T^{*}$.

\section{Results of $\eta / n$ and $\eta / s$}

In Fig. 4(a), the plot of Eq. (11) (black) is almost identical to that of Eq. (19) (red), in accordance with some quantitative overestimates. Two reconstructions of the same inhomogeneous measurement by Joseph et al. [16] and Bluhm et al. [20] are respectively shown in blue circles and a green dashed line, where the former uses an iterative method and the latter employs anisotropic fluid dynamics to extract the local shear viscosity. Our black line locates close to the curve of Joseph et al. [16] above $T_{c}$, which is also considered to have some overestimation [20]. However, a robust non-Fermi liquid signal independent of adjustable parameters is still revealed by this curve, where a rapid drop starts above $T_{c}$ instead of at $T_{c}$, which is exhibited by our two curves too. The smooth average of QMC results (orange dots) [22] also shows such a significant drop, but it lies systematically below our curves with only the fit in shape. Similar phenomena have been reported from the particle conductive observations in two-terminal configurations $[47,48]$, where unexpected enhancements occur before the superfluid transition, which also demonstrates the effects of preformed pairs [49]. Both of the reconstructions shown in the figure have systematic errors (not plotted here), and the approach in the work of Bluhm et al. [20] only uses data where the whole cloud is in the normal phase, so the sensitivity to $\eta$ decreases as $T \rightarrow T_{c}$, which may obscure the phenomenon of a rapid drop above $T_{c}$. Perhaps a future "pseudo-two-fluid" dynamics in the anomalous normal phase with $n_{\text {eff }}$ and $n-n_{\text {eff }}$ as the new effective two-fluid components will provide further understanding, the concept of which has been proposed at the end of Sec. III A.

Previous microscopic calculations of $\eta$ have some difficulties at either low [25] or high temperatures [17,26], and the disagreement near $T_{c}$ seems incompatible [dark yellow, short dashed line and magenta dash-dotted line in Fig. 4(a)]. 

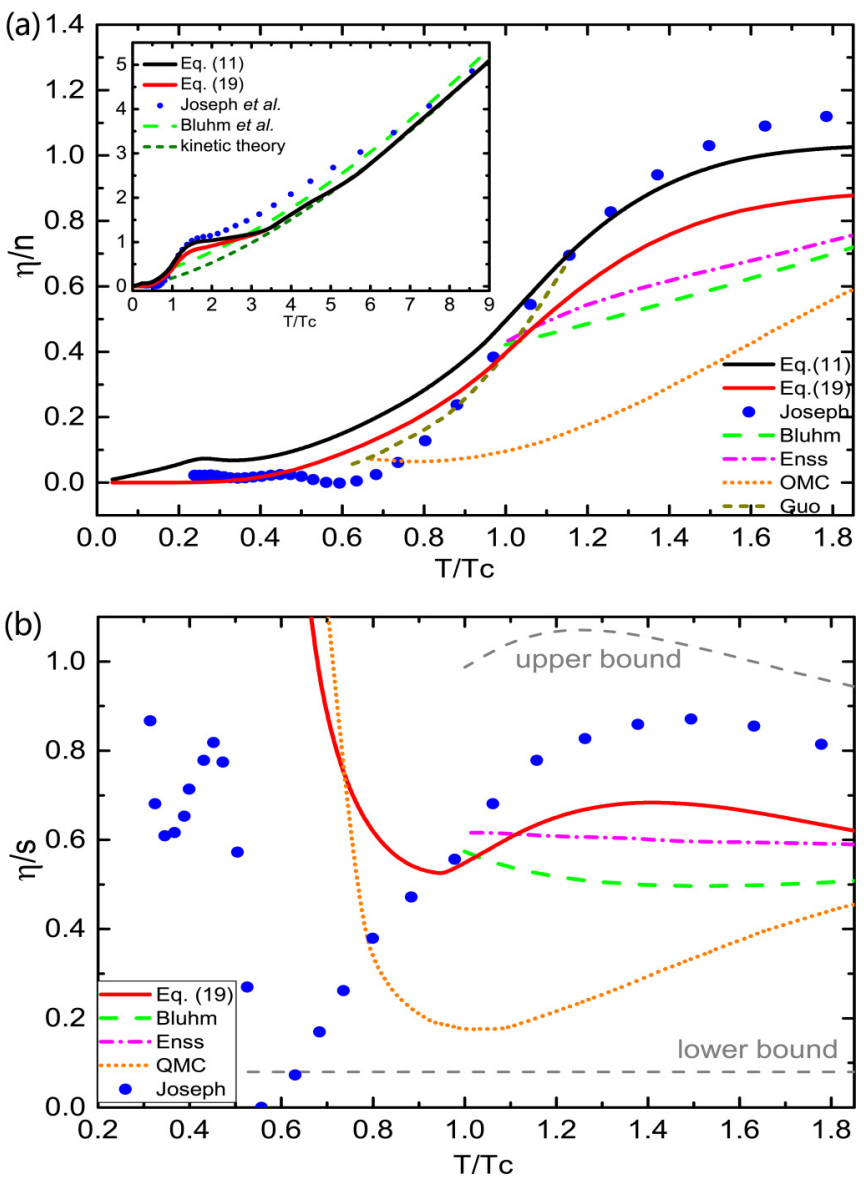

FIG. 4. Shear viscosity as a function of reduced temperature in units of (a) particle density and (b) entropy density. Our calculations of Eqs. (11) and (19) are shown in black and red lines, respectively. For comparison, we show the experimental reconstructions from Joseph et al. [16] (blue circles) and Bluhm et al. [20] (green dashed line), and other theoretical results from Guo et al. [26] (dark yellow short dashed line) around $T_{c}$, Enss et al. [25] (magenta dash-dotted line) at $T>T_{c}$, as well as the QMC result from Wlazlowski et al. [22] (orange dots). The inset of (a) shows a wide temperature regime, where the kinetic result [17] $\eta / n=15(m T)^{3 / 2} /(32 \sqrt{\pi})$ is shown in olive dashed line. The gray dashed lines on the bottom and top of (b) indicate the lower and upper bounds of $\eta / s[10,11]$.

Our Kubo result lies between them and join smoothly from zero to kinetic limits, as shown in the inset of Fig. 4(a). The high- $T$ results almost coincide with the kinetic temperature dependence $\eta / n=15(m T)^{3 / 2} /(32 \sqrt{\pi})$ [17]. The Kubo curve agrees well with the previous theory [26] from the currentcurrent correlation function, also based on the pseudogap model, and the viscosity-independent fitting parameter in their work is naturally fixed in our calculations. In addition, the discrepancies in the pseudogap regime between our results and the theory based on the self-consistent $(G G) G$ scheme [25] come from the different $t$-matrix approximations with different versions: in order to approximately approach the anomalous pseudogap regime, the $\left(G G_{0}\right) G_{0}$ scheme starts from the properties of low-temperature superfluids, while the $(G G) G$ scheme starts from the high-temperature normal fermions.
Figure 4(b) shows $\eta$ in units of entropy density $s$ to compare with a wider class of theories. Applications of holographic duality predict certain universalities of $\eta / s$, such as the famous KSS lower bound $\eta / s \geqslant 1 /(4 \pi)$ [10] and a newly proposed upper bound $\eta / s \lesssim T \tau$ from diffusivity [11]. Our red line has a minimum $\simeq 0.52$, about six times higher than the KSS bound. More significantly, this line has a similar temperature dependence to the upper bound, with about 1.5 times lower, $\eta / s \simeq 2 T \tau / 3$. The entropy density we used is from the MIT experiment [50]. Our results not only verify the unproved upper bound in the UFGs, but they also demonstrate the robustness between $\tau$ and transport coefficients in a systematic way.

\section{CONCLUSIONS AND OUTLOOK}

In conclusion, we have developed an extensible theoretical method that enables study of the scattering mechanisms during thermalization. We have presented a three-fluid dynamics that contains fermionic and bosonic excitations and is consistent with the microscopic pseudogap model, through which the scattering time $\tau$ is calculated by opening almost all scattering channels both for two elementary excitations of fermions and composite bosons, and for an elementary excitation of fermions only. We have also obtained two relations of $\eta$ and $\tau$. One is linear with the prefactor of the thermodynamic quantities; another is complex by presenting a Kubo-based expression for $\eta$ on the stress tensor-tensor correlation functions. Our theoretical results fit well with measurements and other theories. And we have verified the newly proposed universal upper bound of $\eta / s$.

Our results have clearly exhibited the contribution of each scattering process to $\eta$ at different temperatures and verified the importance of pairs. By comparing various relaxation processes among different excitations, we obtain deeper insights of the superfluid and pseudogap phases. We conclude that the boson-fermion scattering channel leads to the universal thermalization time behaviors associated with anomalous transport, and the scale invariance of unitary Fermi gases implies broad applications of our results in different strongly correlated quantum systems where exotic relaxation mechanisms arise, ranging from the important materials [6] to dense quark matters with a similar boson-fermion mixed form [51].

Additionally, our method can be used to study thermal relaxation processes and sound diffusivity consisting of momentum and heat, which have been measured in homogeneous traps recently $[4,41]$. The thermal conductivity $\kappa$ of an ultracold Fermi gas in the BCS-BEC crossover shows some interesting phenomena at the pseudogap and superfluid regimes [52]. The sound diffusivity attains a universal constant below $T_{c}$, which may be quantitatively understood within our theoretical framework.

\section{ACKNOWLEDGMENTS}

This work is supported by the State Key Programs of China under Grant No. 2017YFA0304204 and by the National Natural Science Foundation of China under Grant No. 11375045. 


\section{APPENDIX: COLLISION INTEGRALS}

Here we express the various collision integrals to access relaxation processes for the elementary components $n_{f}$ and $n_{b_{1}}$, which is directly applicable in analogous physical systems with composite bosons and fermions. The collision integral terms of mutual scattering between the same components $C_{f f}[F]$ and $C_{b_{1} b_{1}}[B]$ have been well studied in early work [29,53-55]. They only differ in the distribution functions with the following forms:

$$
\begin{aligned}
C_{f f}[\mathcal{F}]= & \frac{1}{\pi^{3} m_{f}^{2}} \int d \mathbf{p}_{1} \int d \mathbf{p}_{2} \int d \mathbf{p}_{3} \int d \mathbf{p}_{4} \frac{d \sigma_{f}}{d \Omega} \delta\left(\mathbf{p}_{1}+\mathbf{p}_{2}-\mathbf{p}_{3}-\mathbf{p}_{4}\right) \delta\left(\varepsilon_{\mathbf{p}_{1}}+\varepsilon_{\mathbf{p}_{2}}-\varepsilon_{\mathbf{p}_{3}}-\varepsilon_{\mathbf{p}_{4}}\right) \\
& \times\left[\delta\left(\mathbf{k}-\mathbf{p}_{1}\right)+\delta\left(\mathbf{k}-\mathbf{p}_{2}\right)-\delta\left(\mathbf{k}-\mathbf{p}_{3}\right)-\delta\left(\mathbf{k}-\mathbf{p}_{4}\right)\right]\left[(1-F)\left(1-F_{2}\right) F_{3} F_{4}-F F_{2}\left(1-F_{3}\right)\left(1-F_{4}\right)\right], \\
C_{b_{1} b_{1}}[\mathcal{F}]= & \frac{1}{\pi^{3} m_{b}^{2}} \int d \mathbf{q}_{1} \int d \mathbf{q}_{2} \int d \mathbf{q}_{3} \int d \mathbf{q}_{4} \frac{d \sigma_{b}}{d \Omega} \delta\left(\mathbf{q}_{1}+\mathbf{q}_{2}-\mathbf{q}_{3}-\mathbf{q}_{4}\right) \delta\left(\varepsilon_{\mathbf{q}_{1}}+\varepsilon_{\mathbf{q}_{2}}-\varepsilon_{\mathbf{q}_{3}}-\varepsilon_{\mathbf{q}_{4}}\right) \\
& \times\left[\delta\left(\mathbf{k}-\mathbf{q}_{1}\right)+\delta\left(\mathbf{k}-\mathbf{q}_{2}\right)-\delta\left(\mathbf{k}-\mathbf{q}_{3}\right)-\delta\left(\mathbf{k}-\mathbf{q}_{4}\right)\right]\left[\left(1+B_{1}\right)\left(1+B_{2}\right) B_{3} B_{4}-B_{1} B_{2}\left(1+B_{3}\right)\left(1+B_{4}\right)\right] .
\end{aligned}
$$

We denote $\mathbf{k}=\mathbf{p}, \mathbf{q}$, and $F_{i} \equiv F\left(\mathbf{p}_{i}, t\right), B_{i} \equiv B\left(\mathbf{q}_{i}, t\right)$ represent the actual Fermi or Bose distribution function with $i=1,2,3,4$. Since each kind of scattering is considered separately, their differential cross sections are in the vacuum form to avoid double counting: $\frac{d \sigma_{b, f}}{d \Omega}=\frac{a_{b, f}^{2}}{1+p_{r}^{2} a_{b, f}^{2}}$, where $p_{r}$ is the relative momentum of colliding particles, and the subscript $(b, f)$ presents the corresponding scattering processes with scattering lengths of $a_{b}, a_{f}$ and $a_{f b}$, respectively.

We show other collision integrals below:

$$
\begin{aligned}
C_{f b_{0}}[\mathcal{F}]= & \frac{8 n_{b_{0}}}{m_{f b}^{2}} \int d \mathbf{p}_{1} \int d \mathbf{p}_{2} \int d \mathbf{q}_{1} \frac{d \sigma_{f b}}{d \Omega} \delta\left(\mathbf{p}_{1}-\mathbf{p}_{2}-\mathbf{q}_{1}\right) \delta\left(\varepsilon_{b_{0}}+\varepsilon_{\mathbf{p}_{1}}-\varepsilon_{\mathbf{p}_{2}}-\varepsilon_{\mathbf{q}_{1}}\right) \\
& \times\left[\delta\left(\mathbf{k}-\mathbf{p}_{1}\right)-\delta\left(\mathbf{k}-\mathbf{p}_{2}\right)-\delta\left(\mathbf{k}-\mathbf{q}_{1}\right)\right]\left[\left(1-F_{1}\right) F_{2} B_{1}-F_{1}\left(1-F_{2}\right)\left(1+B_{1}\right)\right] \\
C_{f b_{0}}^{\prime}[\mathcal{F}]= & \frac{n_{b_{0}}}{\pi^{3} m_{f b}^{2}} \int d \mathbf{p}_{1} \int d \mathbf{p}_{2} \int d \mathbf{p}_{3} \int d \mathbf{p}_{4} \frac{d \sigma_{f b}}{d \Omega} \delta\left(\mathbf{p}_{1}-\mathbf{p}_{2}-\mathbf{q}\right) \delta\left(\mathbf{q}-\mathbf{p}_{3}-\mathbf{p}_{4}\right) \\
\times & \delta\left(\varepsilon_{b_{0}}+\varepsilon_{\mathbf{p}_{1}}-\varepsilon_{\mathbf{p}_{2}}-\varepsilon_{\mathbf{p}_{3}}-\varepsilon_{\mathbf{p}_{4}}\right)\left[\delta\left(\mathbf{k}-\mathbf{p}_{1}\right)-\delta\left(\mathbf{k}-\mathbf{p}_{2}\right)-\delta\left(\mathbf{k}-\mathbf{p}_{3}\right)-\delta\left(\mathbf{k}-\mathbf{p}_{4}\right)\right] \\
\times & {\left[\left(1-F_{1}\right) F_{2} F_{3} F_{4}-F_{1}\left(1-F_{2}\right)\left(1-F_{3}\right)\left(1-F_{4}\right)\right] } \\
C_{f b_{1}}[\mathcal{F}]= & \frac{1}{\pi^{3} m_{f b}^{2}} \int d \mathbf{p}_{1} \int d \mathbf{q}_{1} \int d \mathbf{p}_{2} \int d \mathbf{q}_{2} \frac{d \sigma_{f b}}{d \Omega} \delta\left(\mathbf{p}_{1}+\mathbf{q}_{1}-\mathbf{p}_{2}-\mathbf{q}_{2}\right) \\
& \times \delta\left(\varepsilon_{\mathbf{p}_{1}}+\varepsilon_{\mathbf{q}_{1}}-\varepsilon_{\mathbf{p}_{2}}-\varepsilon_{\mathbf{q}_{2}}\right)\left[\delta\left(\mathbf{k}-\mathbf{p}_{1}\right)+\delta\left(\mathbf{k}-\mathbf{q}_{1}\right)-\delta\left(\mathbf{k}-\mathbf{p}_{2}\right)-\delta\left(\mathbf{k}-\mathbf{q}_{2}\right)\right] \\
& \times\left[\left(1-F_{1}\right)\left(1+B_{1}\right) F_{2} B_{2}-F_{1} B_{1}\left(1-F_{2}\right)\left(1+B_{2}\right)\right], \\
C_{f b_{1}}^{\prime}[\mathcal{F}]= & \frac{1}{8 \pi^{6} m_{f b}^{2}} \int d \mathbf{p}_{1} \int d \mathbf{q}_{1} \int d \mathbf{p}_{2} \int d \mathbf{p}_{3} \int d \mathbf{p}_{4} \frac{d \sigma_{f b}}{d \Omega} \delta\left(\mathbf{p}_{1}+\mathbf{q}_{1}-\mathbf{p}_{2}-\mathbf{q}_{2}\right) \delta\left(\mathbf{q}_{2}-\mathbf{p}_{3}-\mathbf{p}_{4}\right) \\
\times[\delta(\mathbf{k} & \left.\left.-\mathbf{p}_{1}\right)+\delta\left(\mathbf{k}-\mathbf{q}_{1}\right)-\delta\left(\mathbf{k}-\mathbf{p}_{2}\right)-\delta\left(\mathbf{k}-\mathbf{p}_{3}\right)-\delta\left(\mathbf{k}-\mathbf{p}_{4}\right)\right] \\
\times & \times\left(\varepsilon_{\mathbf{q}_{1}}+\varepsilon_{\mathbf{p}_{1}}-\varepsilon_{\mathbf{p}_{2}}-\varepsilon_{\mathbf{p}_{3}}-\varepsilon_{\mathbf{p}_{4}}\right)\left[\left(1-F_{1}\right)\left(1+B_{1}\right) F_{2} F_{3} F_{4}-F_{1} B_{1}\left(1-F_{2}\right)\left(1-F_{3}\right)\left(1-F_{4}\right)\right], \\
& 8 n_{b_{0}} \int d \mathbf{q}_{1} \int d \mathbf{q}_{2} \int d \mathbf{q}_{3} \frac{d \sigma_{b}}{d \Omega} \delta\left(\mathbf{q}_{1}-\mathbf{q}_{2}-\mathbf{q}_{3}\right) \delta\left(\varepsilon_{b_{0}}+\varepsilon_{\mathbf{q}_{1}}-\varepsilon_{\mathbf{q}_{2}}-\varepsilon_{\mathbf{q}_{3}}\right) \\
C_{b_{1} b_{0}}[\mathcal{F}]= & \left.\left.\times \mathbf{q}_{f b}\right)-\delta\left(\mathbf{k}-\mathbf{q}_{2}\right)-\delta\left(\mathbf{k}-\mathbf{q}_{3}\right)\right]\left[\left(1+B_{1}\right) B_{2} B_{3}-B_{1}\left(1+B_{2}\right)\left(1+B_{3}\right)\right]
\end{aligned}
$$

The trial function $\Psi$ is chosen as the general form for shear viscosity $\Psi=u_{v} u_{\bar{v}}-\delta_{\nu \bar{v}} u^{2} / 3$, where $u_{v, \bar{v}}$ are the components of velocity, $\mathbf{u}=\mathbf{p} / m_{f}\left(\mathbf{q} / m_{b}\right)$. Sequentially, the linearized collision terms become $C_{j l}[\mathcal{F}]=C_{j l}[\delta \mathcal{F}]$ and $C_{j l}^{\prime}[\mathcal{F}]=$ $C_{j l}^{\prime}[\delta \mathcal{F}]$, with the transport index $j=\left(f, b_{1}\right)$ and the component index $l=\left(f, b_{0}, b_{1}\right)$. All of the collision terms are proportional to the trial function $\Psi_{i}$ of the $i$ th scattering particle, and under the relaxation time approximation the relaxation rates in Eqs. (6) and (7) can be obtained by some multiple integrals numerically.
[1] T. Schäfer, Fluid dynamics and viscosity in strongly correlated fluids, Annu. Rev. Nucl. Part. Sci. 64, 125 (2014).
[2] A. Adams, L. D. Carr, T. Schäfer, P. Steinberg, and J. E. Thomas, Strongly correlated quantum fluids: Ultracold 
quantum gases, quantum chromodynamic plasmas, and holographic duality, New J. Phys. 14, 115009 (2012).

[3] C. Cao, E. Elliott, J. Joseph, H. Wu, J. Petricka, T. Schäfer, and J. E. Thomas, Universal quantum viscosity in a unitary Fermi gas, Science 331, 58 (2011).

[4] P. B. Patel, Z-J Yan, B. Mukherjee, R. J. Fletcher, J. Struck, and M. W. Zwierlein, Universal sound diffusion in a strongly interacting Fermi gas, arXiv:1909.02555.

[5] A. Sommer, M. Ku, G. Roati, and M. W. Zwierlein, Universal spin transport in a strongly interacting Fermi gas, Nature (London) 472, 201 (2011).

[6] J. A. N. Bruin, H. Sakai, R. S. Perry, and A. P. Mackenzie, Similarity of scattering rates in metals showing $T$-linear resistivity, Science 339, 804 (2013).

[7] S. Krinner, D. Stadler, D. Husmann, J.-P. Brantut, and T. Esslinger, Observation of quantized conductance in neutral matter, Nature (London) 517, 64 (2015).

[8] S. A. Hartnoll, Theory of universal incoherent metallic transport, Nat. Phys. 11, 54 (2015).

[9] M. Blake, Universal Charge Diffusion and the Butterfly Effect in Holographic Theories, Phys. Rev. Lett. 117, 091601 (2016).

[10] P. K. Kovtun, D. T. Son, and A. O. Starinets, Viscosity in Strongly Interacting Quantum Field Theories from Black Hole Physics, Phys. Rev. Lett. 94, 111601 (2005).

[11] T. Hartman, S. A. Hartnoll, and R. Mahajan, Upper Bound on Diffusivity, Phys. Rev. Lett. 119, 141601 (2017).

[12] G. C. Strinati, P. Pieri, G. Röpke, P. Schuck, and M. Urban, The BCS-BEC crossover: From ultra-cold Fermi gases to nuclear systems, Phys. Rep. 738, 1 (2018).

[13] Q. J. Chen, J. Stajic, S. Tanb, and K. Levin, BCS-BEC crossover: From high temperature superconductors to ultracold superfluid, Phys. Rep. 412, 1 (2005).

[14] C. Cao, E. Elliott, H. Wu, and J. E. Thomas, Searching for perfect fluids: Quantum viscosity in a universal Fermi gas, New J. Phys. 13, 075007 (2011).

[15] E. Elliott, J. A. Joseph, and J. E. Thomas, Anomalous Minimum in the Shear Viscosity of a Fermi Gas, Phys. Rev. Lett. 113, 020406 (2014).

[16] J. A. Joseph, E. Elliott, and J. E. Thomas, Shear Viscosity of a Universal Fermi Gas near the Superfluid Phase Transition, Phys. Rev. Lett. 115, 020401 (2015).

[17] G. M. Bruun and H. Smith, Shear viscosity and damping for a Fermi gas in the unitarity limit, Phys. Rev. A 75, 043612 (2007).

[18] G. Rupak and T. Schäfer, Shear viscosity of a superfluid Fermi gas in the unitarity limit, Phys. Rev. A 76, 053607 (2007).

[19] M. Mannarelli, C. Manuel, and L. Tolos, Phonon contribution to the shear viscosity of a superfluid Fermi gas in the unitarity limit, Ann. Phys. 336, 12 (2013).

[20] M. Bluhm, J. X. Hou, and T. Schäfer, Determination of the Density and Temperature Dependence of the Shear Viscosity of a Unitary Fermi Gas Based on Hydrodynamic Flow, Phys. Rev. Lett. 119, 065302 (2017).

[21] G. Wlazłowski, P. Magierski, and J. E. Drut, Shear Viscosity of a Unitary Fermi Gas, Phys. Rev. Lett. 109, 020406 (2012).

[22] G. Wlazłowski, P. Magierski, A. Bulgac, and K. J. Roche, Temperature evolution of the shear viscosity in a unitary Fermi gas, Phys. Rev. A 88, 013639 (2013).

[23] G. Wlazłowski, W. Quan, and A. Bulgac, Perfect-fluid behavior of a dilute Fermi gas near unitary, Phys. Rev. A 92, 063628 (2015).
[24] E. Taylor and M. Randeria, Viscosity of strongly interacting quantum fluids: Spectral functions and sum rules, Phys. Rev. A 81, 053610 (2010).

[25] T. Enss, R. Haussmann, and W. Zwerger, Viscosity and scale invariance in the unitary Fermi gas, Ann. Phys. 326, 770 (2011).

[26] H. Guo, D. Wulin, C.-C. Chien, and K. Levin, Microscopic Approach to Shear Viscosities of Unitary Fermi Gases above and below the Superfluid Transition, Phys. Rev. Lett. 107, 020403 (2011).

[27] H. Guo, D. Wulin, C.-C. Chien, and K. Levin, Perfect fluids and bad metals: Insights from ultracold Fermi gases, New J. Phys. 13, 075011 (2011).

[28] J. Hofmann, High-temperature expansion of the viscosity in interacting quantum gases, Phys. Rev. A 101, 013620 (2020).

[29] P. Massignan, G. M. Bruun, and H. Smith, Viscous relaxation and collective oscillations in a trapped Fermi gas near the unitarity limit, Phys. Rev. A 71, 033607 (2005).

[30] G. M. Bruun and H. Smith, Viscosity and thermal relaxation for a resonantly interacting Fermi gas, Phys. Rev. A 72, 043605 (2005).

[31] K. Dusling and T. Schäfer, Bulk Viscosity and Conformal Symmetry Breaking in the Dilute Fermi Gas near Unitarity, Phys. Rev. Lett. 111, 120603 (2013).

[32] T. Enss, Bulk Viscosity and Contact Correlations in Attractive Fermi Gases, Phys. Rev. Lett. 123, 205301 (2019).

[33] Y. Sagi, T. E. Drake, R. Paudel, R. Chapurin, and D. S. Jin, Breakdown of the Fermi Liquid Description for Strongly Interacting Fermions, Phys. Rev. Lett. 114, 075301 (2015).

[34] J. P. Gaebler, J. T. Stewart, T. E. Drake, D. S. Jin, A. Perali, P. Pieri, and G. C. Strinati, Observation of pseudogap behavior in a strongly interacting Fermi gas, Nat. Phys. 6, 569 (2010).

[35] H. Guo, W. M. Cai, Y. He, and C.-C. Chien, Relation connecting thermodynamics and transport of atomic unitary Fermi superfluid, Phys. Rev. A 95, 033638 (2017).

[36] H. Dong, W. Y. Zhang, L. Zhou, and Y. L. Ma, Transition and damping of collective modes in a trapped Fermi gas between BCS and unitary limits near the phase transition, Sci. Rep. 5, 15848 (2015).

[37] S. Nascimbène, N. Navon, K. J. Jiang, F. Chevy, and C. Salomon, Exploring the thermodynamics of a universal Fermi gas, Nature (London) 463, 1057 (2010).

[38] L. A. Sidorenkov, M. K. Tey, R. Grimm, Y.-H. Hou, L. Pitaevskii, and S. Stringari, Second sound and the superfluid fraction in a Fermi gas with resonant interactions, Nature (London) 498, 78 (2013).

[39] P. Zhang and Z. Yu, Energy-absorption spectroscopy of unitary Fermi gases in a uniform potential, Phys. Rev. A 97, 041601(R) (2018).

[40] H. Hu, P. Zou, and X. J. Liu, Low-momentum dynamic structure factor of a strongly interacting Fermi gas at finite temperature: A two-fluid hydrodynamic description, Phys. Rev. A 97, 023615 (2018).

[41] L. Baird, X. Wang, S. Roof, and J. E. Thomas, Measuring the Hydrodynamic Linear Response of a Unitary Fermi Gas, Phys. Rev. Lett. 123, 160402 (2019).

[42] Y. Inada, M. Horikoshi, S. Nakajima, M. K. Gonokami, M. Ueda, and T. Mukaiyama, Critical Temperature and Condensate Fraction of a Fermion Pair Condensate, Phys. Rev. Lett. 101, 180406 (2008). 
[43] S. Hoinka, P. Dyke, M. G. Lingham, J. J. Kinnunen, G. M. Bruun, and C. J. Vale, Goldstone mode and pair-breaking excitations in atomic Fermi superfluids, Nat. Phys. 13, 943 (2017).

[44] R. Lopes, C. Eigen, N. Navon, D. Clément, R. P. Smith, and Z. Hadzibabic, Quantum Depletion of a Homogeneous BoseEinstein Condensate, Phys. Rev. Lett. 119, 190404 (2017).

[45] Q. J. Chen, Y. He, C.-C. Chien, and K. Levin, Theory of radio frequency spectroscopy experiments in ultracold Fermi gases and their relation to photoemission in the cuprates, Rep. Prog. Phys. 72, 122501 (2009).

[46] I. Kosztin, Q. J. Chen, Y.-J. Kao, and K. Levin, Pair excitations, collective modes, and gauge invariance in the BCS-BoseEinstein crossover scenario, Phys. Rev. B 61, 11662 (2000).

[47] S. Krinner, M. Lebrat, D. Husmann, C. Grenier, J.-P. Brantut, and T. Esslinger, Mapping out spin and particle conductances in a quantum point contact, Proc. Natl. Acad. Sci. USA 113, 8144 (2016).

[48] D. Stadler, S. Krinner, J. Meineke, J.-P. Brantut, and T. Esslinger, Observing the drop of resistance in the flow of a superfluid Fermi gas, Nature (London) 491, 736 (2012).
[49] S. Uchino and M. Ueda, Anomalous Transport in the Superfluid Fluctuation Regime, Phys. Rev. Lett. 118, 105303 (2017).

[50] M. J. H. Ku, A. T. Sommer, L. W. Cheuk, and M. W. Zwierlein, Revealing the superfluid Lambda transition in the universal thermodynamics of a unitary Fermi gas, Science 335, 563 (2012).

[51] K. Maeda, G. Baym, and T. Hatsuda, Simulating Dense QCD with Ultracold Atomic Boson-Fermion Mixtures, Phys. Rev. Lett. 103, 085301 (2009).

[52] H. Zhou and Y. L. Ma, Thermal conductivity of an ultracold Fermi gas in the BCS-BEC crossover (unpublished).

[53] T. Nikuni and A. Griffin, Hydrodynamic damping in trapped Bose gases, J. Low Temp. Phys. 111, 793 (1998).

[54] T. Nikuni and A. Griffin, Landau-Khalatnikov two-fluid hydrodynamics of a trapped Bose gas, Phys. Rev. A 63, 033608 (2001).

[55] G. M. Kavoulakis, C. J. Pethick, and H. Smith, Collisional relaxation in diffuse clouds of trapped bosons, Phys. Rev. A 61, 053603 (2000). 